\title{
盐湖卤水及盐类矿物的氯同位素组成 *
}

\author{
肖应凯 刘卫国 周引民 孙大鹏 \\ (中国科学院青海盐湖研究所, 西宁 810008)
}

\section{关键词氞位素 盐湖卤水 盐类矿物}

自然界氯有两种稳定同位素 ${ }^{35} \mathrm{Cl}$ 和 ${ }^{37} \mathrm{Cl}$, 过去建立了两种测定氯同位素组成的方法. Owen ${ }^{[1]}$, Kaufmann ${ }^{[2]}$ 和 Long ${ }^{[3]}$ 采用测量由 $\mathrm{CH}_{3} \mathrm{Cl}$ 电离产生的 $\mathrm{CH}_{3} \mathrm{Cl}^{+}$离子来测量氯同位素, 测量精度已高达 $0.009 \%$. ${ }^{[3]}$. 与之相比, 由 Shields ${ }^{[4]}$ 和 Vengosh ${ }^{[5]}$ 等论述的 $\mathrm{Cl}^{-}$负离子热电离 质谱法的测定精度 $(0.2 \%)$ 则要低的多.

最近我们建立了基于 $\mathrm{Cs}_{2} \mathrm{Cl}^{+}$离子测量的新的正热电离质谱测定氯同位素的方法 ${ }^{[6,7]}$, 该 方法现已被 Magenheim ${ }^{[8]}$ 和 Volpe ${ }^{[9]}$ 等用来测定海水和低浓度样品大气气溶胶中的氯同位素 组成, 发现大气气溶胶中 ${ }^{37} \mathrm{Cl}$ 明显地高于对应海水中的 ${ }^{37} \mathrm{Cl}$. 本实验采用改进后的 $\mathrm{Cs}_{2} \mathrm{Cl}^{+}$正 热电离质谱法, 对一些来自不同地区的盐类矿物以及柴达木盆地盐湖卤水中的氯同位素组成 进行了研究, 发现了氯同位素组成的一些变化规律.

\section{1. 样品制备}

本工作从不同渠道收集到国内外一些地区的盐类沉积矿床的盐类矿物. 青海柴达木盆地 盐湖卤水样品, 这些样品氯含量均很高, 特别是盐湖卤水则是氯化物的饱和溶液, 因此这些样 品的氯同位素基本上反映了高盐度体系的氯同位素分布特征.

盐湖卤水可直接取样, 盐类矿物可加适量水溶解. 为了减少样品不均匀性带来的影响, 可 适当增加固体样品的取样量, 以上溶液将采用离子交换的方法分离氯 ${ }^{[10]}$, 含氯的溶液 (以 $\mathrm{HCl}$ 形式) 用 $\mathrm{Cs}_{2} \mathrm{CO}_{3}$ 溶液调节 $\mathrm{pH}$ 为 3 , 其氯的浓度为 $5 \sim 10 \mathrm{mg} / \mathrm{mL}$.

\section{2 质谱测定过程}

采用石墨涂样技术进行氯同位素测定 ${ }^{[6,7]}$. 取适量(约 $10 \mu \mathrm{g}$ 氯)交换分离后含氯的样品 液, 涂于事先涂有约 $100 \mu \mathrm{g}$ 碳的石墨悬浮液的钽带上, 通 $1.1 \mathrm{~A}$ 电流加热 $3 \mathrm{~min}$ 使溶液蒸干, 装入质谱计. 测量时在计算机控制下采用峰跳扫交替测量 ${ }^{133} \mathrm{Cs}_{2}{ }^{35} \mathrm{Cl}^{+}$和 ${ }^{133} \mathrm{Cs}_{2}{ }^{37} \mathrm{Cl}^{+}(m / Q$ 分 别为 301 和 303) 离子流强度, 并直接给出 ${ }^{37} \mathrm{Cl} /{ }^{35} \mathrm{Cl}$ 比值. 本实验对由 Shields ${ }^{[4]}$ 论述的氯同位 素标准样品 NIST SRM975 进行了重复测定, 结果列于表 1,9 次涂样测定的平均 ${ }^{37} \mathrm{Cl} /{ }^{35} \mathrm{Cl}$ 值 为 $0.31893 \pm 0.00003(1 \sigma)$, 测定精度为 $0.009 \%$.

1995-12-26 收稿, 1996-04-22 收修改稿

* 国家自然科学基金资助项目 
表 1 SRM975 重复测定结果

\begin{tabular}{cccc}
\hline 次数 & 带电流/A & $\mathrm{Cs}_{2}{ }^{37} \mathrm{Cl}^{+} / 10^{-12} \mathrm{~A}$ & ${ }^{37} \mathrm{Cl} /{ }^{35} \mathrm{Cl}$ \\
\hline 1 & 1.146 & 6.7 & $0.31887 \pm 0.00002$ \\
2 & 1.112 & 6.9 & $0.31895 \pm 0.00004$ \\
3 & 1.111 & 6.3 & $0.31907 \pm 0.00006$ \\
4 & 1.123 & 6.7 & $0.31888 \pm 0.00003$ \\
5 & 1.103 & 6.9 & $0.31905 \pm 0.00005$ \\
6 & 1.128 & 6.6 & $0.31893 \pm 0.00005$ \\
7 & 1.284 & 6.2 & $0.31893 \pm 0.00004$ \\
8 & 1.210 & 6.8 & $0.31887 \pm 0.00003$ \\
9 & 1.099 & 6.6 & $0.31886 \pm 0.00006$ \\
平均值 & & & $0.31893 \pm 0.00003$ \\
精度 $(1 \sigma)$ & & & $0.009 \%$ \\
\hline
\end{tabular}

\section{3 结果与讨论}

\section{1 盐湖卤水中的氮同位素组成}

柴达木盆地是我国盐湖集中分布区, 由于此地区气候干燥, 降雨量稀少, 大部分盐湖已是 $\mathrm{NaCl}$ 的饱和溶液, 平均盐度已高达 $332 \mathrm{~g} / \mathrm{L}$, 是海水的 10 倍. 由于受到强烈的蒸发, 湖底已有 大量的石盐、石音和芒硝等盐类沉积, 这些卤水的 ${ }^{37} \mathrm{Cl} /{ }^{35} \mathrm{Cl}$ 值的测定结果列于表 2 , 平均

表 2 柴达木盆地盐湖囟水氯同位素比值

\begin{tabular}{|c|c|c|c|c|c|}
\hline & 盐湖名称 & $\mathrm{pH}$ & 比重 & 埭含量 $/ \mathrm{g} \cdot \mathrm{L}^{-1}$ & ${ }^{37} \mathrm{Cl} /{ }^{35} \mathrm{Cl}$ \\
\hline 1 & 青 海 湖 & 8.90 & 1.011 & 6.00 & $0.31912 \pm 0.00014$ \\
\hline 2 & 希里沟湖 & 8.20 & 1.040 & 3.00 & $0.31910 \pm 0.00015$ \\
\hline 3 & 尔 海 & 8.22 & 1.058 & & $0.31909 \pm 0.00010$ \\
\hline 4 & 托 苏 湖 & 8.90 & 1.022 & 12.0 & $0.31903 \pm 0.00004$ \\
\hline 5 & 大苏干湖 & 8.88 & 1.021 & 9.00 & $0.31922 \pm 0.00006$ \\
\hline 6 & 小苏干湖 & 8.64 & 1.013 & 6.00 & $0.31917 \pm 0.00007$ \\
\hline 7 & 冷湖 & 7.76 & 1.002 & 3.00 & $0.31942 \pm 0.00007$ \\
\hline 8 & 茶 卡 湖 & 7.31 & 1.052 & 45.0 & $0.31915 \pm 0.00008$ \\
\hline 9 & 小柴达木湖 & 8.14 & 1.126 & 83.0 & $0.31941 \pm 0.00015$ \\
\hline 10 & 柯 柯 湖 & 7.25 & 1.211 & 185 & $0.31906 \pm 0.00012$ \\
\hline 11 & 柴 凯 湖 & 7.65 & 1.158 & 136 & $0.31899 \pm 0.00023$ \\
\hline 12 & 达布逊湖 & 6.80 & 1.233 & & $0.31893 \pm 0.00012$ \\
\hline 13 & 达 西 湖 & 6.07 & 1.282 & & $0.31872 \pm 0.00011$ \\
\hline 14 & 南八仙(晶) & 7.16 & 1.205 & 190 & $0.31922 \pm 0.00007$ \\
\hline 15 & 巴伦马海湖 & 6.50 & 1.207 & 195 & $0.31925 \pm 0.00011$ \\
\hline 16 & 西台吉乃尔湖 & 7.60 & 1.147 & 120 & $0.31905 \pm 0.00010$ \\
\hline 17 & 东台吉乃尔湖 & 6.99 & 1.266 & 187 & $0.31914 \pm 0.00020$ \\
\hline 18 & 塔尔丁湖 & 7.37 & 1.255 & 124 & $0.31901 \pm 0.00017$ \\
\hline 19 & 大浪滩(晶) & 7.06 & 1.223 & 186 & $0.31899 \pm 0.00008$ \\
\hline 20 & 茫 崖 湖 & 7.49 & 1.250 & 150 & $0.31911 \pm 0.00007$ \\
\hline 21 & 尔斯库勒湖 & 7.10 & 1.109 & 171 & $0.31891 \pm 0.00015$ \\
\hline 22 & 一里坪 (晶) & 7.03 & 1.226 & 195 & $0.31884 \pm 0.00012$ \\
\hline 23 & 昆特依湖 & 6.65 & 1.212 & 199 & $0.31893 \pm 0.00018$ \\
\hline 24 & 别勒傩 (晶) & 5.84 & 1.282 & 240 & $0.31877 \pm 0.00001$ \\
\hline 25 & 协 作 湖 & 5.63 & 1.263 & 252 & $0.31864 \pm 0.00005$ \\
\hline 26 & 牛 郎 湖 & 5.34 & 1.284 & 277 & $0.31861 \pm 0.00011$ \\
\hline 27 & 织 女 湖 & 4.43 & 1.300 & & $0.31859 \pm 0.00021$ \\
\hline 28 & 钾湖 & 4.62 & 1.300 & 293 & $0.31843 \pm 0.00006$ \\
\hline \multicolumn{5}{|c|}{ 咸水湖 $(1 \sim 9)$} & $0.31919 \pm 0.00009(1 \sigma)$ \\
\hline \multicolumn{3}{|c|}{ 平均 ${ }^{37} \mathrm{Cl} /{ }^{30} \mathrm{Cl}$ 比值 } & \multicolumn{2}{|c|}{ 盐湖 $(10 \sim 28)$} & $0.31890 \pm 0.00010(1 \sigma)$ \\
\hline
\end{tabular}


${ }^{37} \mathrm{Cl} /{ }^{35} \mathrm{Cl}$ 值为 $0.31890 \pm 0.00010$, 与卤水的 $\mathrm{pH}$ 值、氯含量等因素有关, 并随卤水 $\mathrm{pH}$ 值的降 低而降低, 这是盐湖卤水受蒸发的结果. 当卤水蒸发时会伴随大量的盐类矿物的析出, 此时卤 水的 $\mathrm{pH}$ 值也随之降低, 而氯含量增高, 由于 ${ }^{37} \mathrm{Cl}$ 富集在固相, 因此造成了卤水 ${ }^{37} \mathrm{Cl} /{ }^{35} \mathrm{Cl}$ 值的降 低.

\section{2 盐类矿物的氯同位素组成}

当盐湖卤水被蒸发到饱和状态时有盐类矿物析出, 其中氯化物是主要的成分之一．有些 盐类沉积由于沉积年代很早, 已形成岩盐, 完全和卤水脱离, 但大部分现代盐类沉积则与卤水 共存, 这些盐类矿物反映了卤水母液的特征. 表 3 列出了来自一些不同地区、不同类型和不同 年代盐类矿物的 ${ }^{37} \mathrm{Cl} /{ }^{35} \mathrm{Cl}$ 测定值, 平均值是 $0.31902 \pm 0.00005$, 其中柴达木盆地巴仑马海盐 湖盐类沉积的 ${ }^{37} \mathrm{Cl} /{ }^{35} \mathrm{Cl}$ 值最高 $(0.31947)$. 总之, 与盐湖卤水相比, 盐类沉积明显地富集 ${ }^{37} \mathrm{Cl}$, 平均同位素分馏系数为 $0.31902 / 0.31890=1.0006$, 与大柴达木湖的结果 ${ }^{[10]}$ 完全一致.

表 3 盐类矿物的氯同位素组成

\begin{tabular}{|c|c|c|c|c|}
\hline & 样品来源 & 矿物类型 & 年代 & ${ }^{37} \mathrm{Cl} /{ }^{35} \mathrm{Cl}$ \\
\hline 1 & 中国, 茶卡 & 石盐 & 现代 & $0.31917 \pm 0.00005$ \\
\hline 2 & 中国, 柯柯 & 石盐 & 现代 & $0.31906 \pm 0.00005$ \\
\hline 3 & 中国,柴凯 & 石盐 & 现代 & $0.31913 \pm 0.00002$ \\
\hline 4 & 中国, 别勒滩 & 石盐 & 现代 & $0.31892 \pm 0.00006$ \\
\hline 5 & 中国, 巴伦马海 & 石盐 & 现代 & $0.31947 \pm 0.00003$ \\
\hline 6 & 中国, 德宗马海 & 石盐 & 现代 & $0.31910 \pm 0.00003$ \\
\hline 7 & 中国, 织女湖 & 石盐 & 现代 & $0.31893 \pm 0.00010$ \\
\hline 8 & 中国, 牛郎湖 & 石盐 & 现代 & $0.31897 \pm 0.00012$ \\
\hline 9 & 中国,钾湖 & 石盐 & 现代 & $0.31909 \pm 0.00003$ \\
\hline 10 & 中国,昆特依 & 石盐 & 现代 & $0.31889 \pm 0.00013$ \\
\hline 11 & 中国,一里坪 & 石盐 & 现代 & $0.31899 \pm 0.00003$ \\
\hline 12 & 中国, 东台 & 石盐 & 现代 & $0.31903 \pm 0.00009$ \\
\hline 13 & 中国,塔尔丁湖 & 石盐 & 现代 & $0.31906 \pm 0.00005$ \\
\hline 14 & 中国, 大浪滩 & 石盐 & 现代 & $0.31919 \pm 0.00005$ \\
\hline 15 & 中国, 尔斯库勒湖 & 石盐 & 现代 & $0.31892 \pm 0.00002$ \\
\hline 16 & 中国,山东 & 盐岩 & 第三纪 & $0.31900 \pm 0.00006$ \\
\hline 17 & 中国, 三水 & 盐岩 & 第三纪 & $0.31900 \pm 0.00003$ \\
\hline 18 & 中国, 塔里木 & 盐岩 & 石炭纪 & $0.31904 \pm 0.00006$ \\
\hline 19 & 中国, 鄂尔多斯 & 盐岩 & 奥陶纪 & $0.31897 \pm 0.00010$ \\
\hline 20 & 中国, 鄂尔多斯 & 盐岩 & 奥陶纪 & $0.31901 \pm 0.00012$ \\
\hline 21 & 中国, 鄂尔多斯 & 盐岩 & 奥陶纪 & $0.31892 \pm 0.00003$ \\
\hline 22 & 中国, 鄂尔多斯 & 盐岩 & 奥陶纪 & $0.31903 \pm 0.00013$ \\
\hline 23 & 中国, 鄂尔多斯 & 盐岩 & 奥陶纪 & $0.31903 \pm 0.00003$ \\
\hline 24 & 俄罗斯 & 钾石盐 & & $0.31912 \pm 0.00009$ \\
\hline 25 & 俄罗斯 & 钾石盐 & & $0.31883 \pm 0.00012$ \\
\hline 26 & 俄罗斯 & 石盐 & & $0.31891 \pm 0.00003$ \\
\hline 27 & 中国, 山东 & 石盐 & 第三纪 & $0.31918 \pm 0.00013$ \\
\hline 28 & 西班牙, 卡塔罗那 & 光卤石 & & $0.31887 \pm 0.00003$ \\
\hline \multirow[t]{2}{*}{29} & 西班牙, 卡塔罗那 & 盐岩 & & $0.31870 \pm 0.00009$ \\
\hline & 平均 & & & $0.31902 \pm 0.00005$ \\
\hline
\end{tabular}


以上结果表明, 由于蒸发结晶过程中 ${ }^{37} \mathrm{Cl}$ 与 ${ }^{35} \mathrm{Cl}$ 之间的氯同位素效应, 使得盐类矿物比盐 湖卤水有较高的 ${ }^{37} \mathrm{Cl} /{ }^{35} \mathrm{Cl}$ 比值, 盐湖攴水的 ${ }^{37} \mathrm{Cl} /{ }^{35} \mathrm{Cl}$ 值反应了卤水蒸发度等盐卤水的水化学 条件的变化, 通过对盐湖卤水氯同位素的研究可以示踪盐湖演化及卤水蒸发的变化过程, 盐类 矿物的氯同位素组成与沉积环境地质条件及盐卤水的氯同位素组成有关,有可能成为研究盐 类矿床沉积地球化学的一个新的手段.

\section{参考文献}

1 Owen H R, Schaefler O A. The isotope abundances from various sources. J Amer Chem Soc, 1955, 77: 898

2 Kaufmann R, Long A, Bentley H et al. Natural chloride variations. Nature, 1984, 309: 211 213

3 Long A, Kaufmann R. High precision measurement of chlorine stable isotopie ratios. Geochim Cosmochim Acta, 1993, 57: 2907

4 Shields W S, Murphy T J, Garner E L et al. Absolute isotopic abundance ratio and the atomic weight of chloride. J Amer Chem Soc, 1962, 84: 1959

5 Vengosh A, Chivas A. Direct determination of boron and chloride isotopic composition in geological materials by negative thermal-ionization mass spectrometry. Chem Geol, 1989, 79: 333

6 Xiao Y K, Zhang C G. High precision isotopic measurement of chloride by thermal-ionization mass spectrometry of $\mathrm{Cs}_{2} \mathrm{Cl}^{+}$ ion. Int J Mass Spectrom Ion Proc, 1992, 116: 183

7 Xiao Y K, Zhou Y M, Liu W G. Precise measurement of chloride isotopes based on $\mathrm{Cs}_{2} \mathrm{Cl}^{+}$by thermal ionization mass spectrometry. Analytical Letters, 1995, 28: 1295

8 Magenheim A J, Spivack A J, Volpe C. Precise determination of stable chloride isotopic ratios in the low concentration natural sample. Geochim et Cosmochim Acta, 1994, 58: 3117

9 Volpe C, Spivack A. Stable chloride isotopic composition of marine aerosol particles in the westen Atlantic Ocean. Geophys Res Lett, 1994, 21: 1161

10 肖应凯, 金 琳, 刘卫国等. 大柴达木湖的氛同位素组成. 科学通报, 1994,39(11): 1319 1322 